\title{
A case of scleroderma presenting as cardiospasm (achalasia of the oesophagus)
}

\author{
S. M. HANNA* \\ M.B., Ch.B., M.R.C.P., D.P.M. \\ Senior Registrar, University Department of Psychiatry, Whiteley Wood Clinic, Sheffield, S10 $3 T L$
}

\section{Introduction}

Scleroderma is a systemic disease which involves the collagenous connective tissue of different organs in the body. The skin lesions are merely the external manifestations of this sytemic disease and are quite frequently the obvious features that lead to the diagnosis of the condition. Visceral changes however, can precede the skin manifestation (Rodnan \& Fennell, 1962). The length of time for which this may be so is not generally recognized. For this reason the following case history is presented and shows that visceral changes can be present for several years

*Present position and address: Clinical Director and Director of Research, Cherry Hospital, Goldsboro, North Carolina, 27530, United States. before skin changes are manifest. It also shows that involvement of the oesophagus presented as cardiospasm (achalasia) and was treated as such for years.

The patient also illustrates the fact that vague abdominal symptoms simulating intestinal obstruction can be an early manifestation of the illness. Finally the case history confirms the statement that oesophageal involvement can be present in patients with Raynaud's phenomenon.

\section{Case report}

The patient was fairly well till the age of 69 when at a routine mass X-ray it was found that she had a grossly dilated oesophagus. The diagnosis of cardiospasm (achalasia) was made. Figures 1 and 2 show a

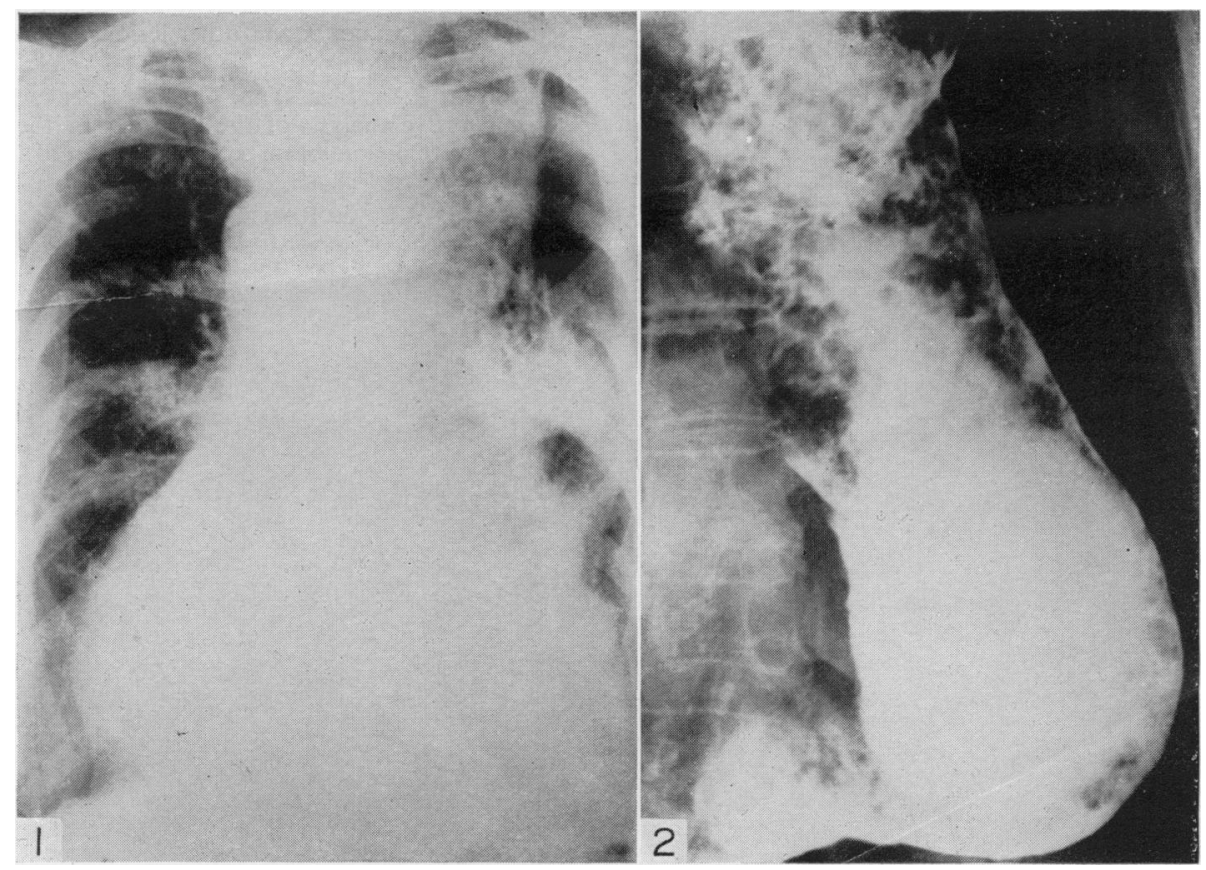

Figs. 1 and 2. Posterio-anterior view and lateral view of a grossly dilated oesophagus. Achalasia (cardiospasm) was diagnosed. Hydrostatic dilation of the cardia gave partial relief. Many years later other manifestations of scleroderma appeared and the diagnosis was made and confirmed on necropsy. 
postero-anterior and a lateral view of the dilated oesophagus. On questioning, the patient said she had had dysphagia and dyspepsia for some time. Hydrostatic dilatation of the cardia relieved the symptoms. Heller's operation was considered, but postponed in view of the relief of her symptoms, and because the patient failed to attend the follow-up clinics. Later she moved to another part of the country.

Six years later she complained of recurrent abdominal pain, poor appetite, increasing constipation and weight loss. She attended the local hospital and was admitted for investigations.

Examination showed a doubtful mass in the epigastrium and no other abnormality. A barium enema was not of much use as it could not be properly retained. It just showed scattered diverticula in the sigmoid colon. A barium meal showed achalasia of the cardia with gross oesophageal dilation. This made examination of the stomach rather unsatisfactory as the barium only entered the stomach in small amounts. However, no definite abnormality was shown in the stomach or duodenum. There was no delay in gastric emptying. The colon was not adequately visualized in the $24-\mathrm{hr}$ film.

Operation. As the X-ray results were not conclusive and because of the clinical picture of subacute intestinal obstruction, an exploratory laparotomy was carried out. The liver was found to be enlarged. The stomach, duodenum and small bowel were all normal. There was diverticulosis of the sigmoid colon, otherwise there was nothing abnormal in the large colon. Even after the barium studies and the laparotomy, scleroderma was not suspected and she was still diagnosed as cardiospasm.

Shortly after the laparotomy the patient's mental state started to deteriorate rapidly. She became noisy, disorientated for time, place and person, her memory for both recent and remote events was very impaired and she required constant supervision. A year after the laparotomy she had to be admitted to a psychiatric hospital, diagnosed as 'senile dementia'. She was 77 years old then. This was when the author saw her. It was then noticed that her face showed the characteristics of scleroderma. The face was mask-like, the skin was smooth, waxy, leathery and tight and it could not be lifted from the deeper structures. There were no sclerodermic or trophic changes in the hands or fingers, but the fingers were blue and a history of Raynaud's phenomenon was obtained from the patient's daughter. From the clinical picture and the long history of visceral involvement, the diagnosis of scleroderma was made. Shortly after her admission the patient developed aspiration pneumonia and died aged 77 . The diagnosis of scleroderma was confirmed at necropsy.
Necropsy. The oesophagus was found to be thickened and distended (Fig. 3). It contained debris and there was a leucoplakia of the squamous epithelium. Histologically muscle hypertrophy and increased fibrous tissue were noted with reduction of the squamous epithelium and there was lymphocytic infiltration especially around the vessels. In the skin there was atrophy of the epidermis and hyalinization of the upper dermis. The dermis itself was very thick and the dermal subcutaneous border irregular. Sweat glands were seen frequently in the dermis. The appearances were those of long-standing systemic scleroderma. The lung showed a cystic sclerosis, or honey-comb change typical of generalized scleroderma. There was a mild nephrosclerosis.

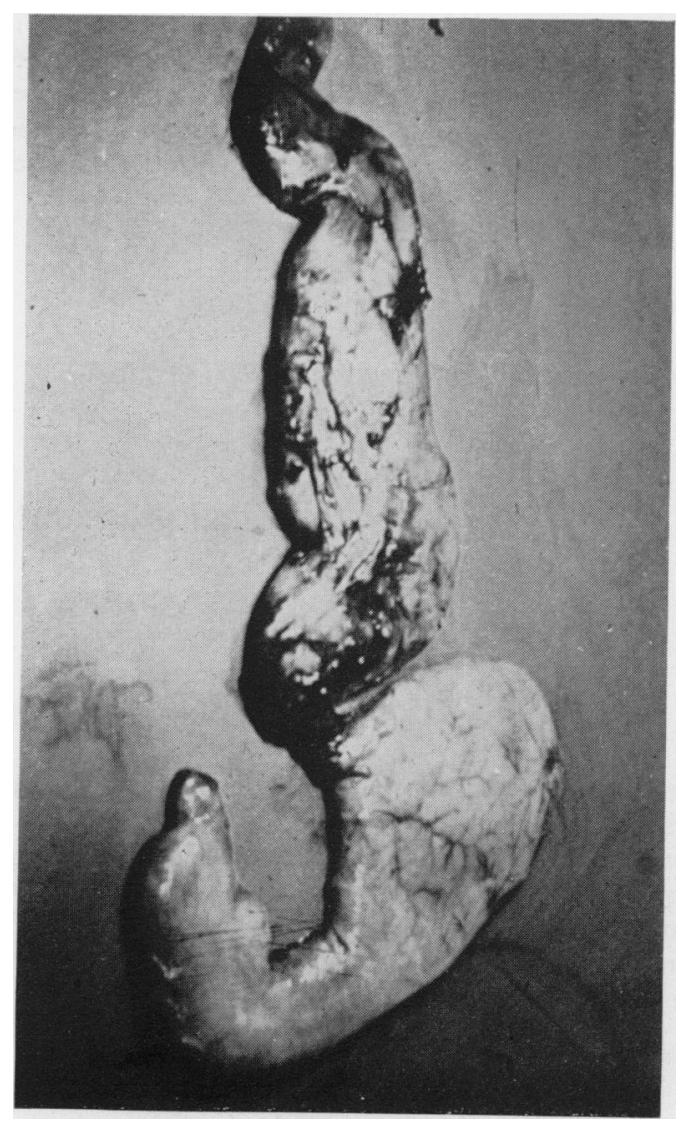

FIG. 3. The dilated oesophagus at necropsy.

\section{Discussion}

It is well known that the gastro-intestinal tract can be extensively involved in scleroderma. The commonest lesion is in the oesophagus and the occurrence of dysphagia has been recognized for more than half a century. The dysphagia may be an early symptom and even precede the skin lesions. In 
most cases of diffuse systemic scleroderma, the onset of the illness is marked by involvement of the viscera and it is well known that scleroderma may be present for many years before the development of clinically detectable visceral disease or it may continue to exist indefinitely with little or no evidence of systemic dysfunction. Not infrequently, however, one encounters patients in whom the occurrence of visceral changes typical of systemic scleroderma antedates the appearance of dermal changes, thus creating as it were a state of progressive systemic sclerosis sine scleroderma as described by Rodnan \& Fennel (1962).

In most such cases this condition is only temporary, typical skin changes becoming apparent within a period of several months to a year. In the patient presented the skin changes became apparent 7 years after the visceral involvement. This is much longer than in any case found in the literature. Even the three patients described by Rodnan \& Fennel (1962) as systemic sclerosis sine scleroderma, died within 3 years of the onset of the visceral changes. The other points of interest are:

(i) That the patient was thought to be suffering and was treated for achalasia for 7 years.

(ii) That involvement of the intestine presented 6 years after the achalasia was diagnosed, as subacute intestinal obstruction and led to laparotomy.

(iii) The presence of Raynaud's phenomenon in this patient is in keeping with the previous observations that involvement of the oesophagus with aperistalsis is more frequent in patients with Raynaud's phenomenon (Stevens et al., 1964).

\section{References}

Rodnan, G.P. \& Fennell, R.H. JR (1962) Progressive systemic sclerosis sine scleroderma. Journal of the American Medical Association, 180, 665.

Stevens, M.B., Hookman, P., Siegel, C.I., Esterly, J.R., Shulman, L.E. \& Hendrix, T.R. (1964) Aperistalsis of the oesophagus in patients with connective tissue disorders and Raynaud's phenomenon. New England Journal of Medicine, 270, 1218.

\title{
Unusual presentation of multiple myeloma
}

\author{
ANDREW G. A. HeFFERnAN* \\ M.D., M.Sc. \\ Mater Misericordiae Hospital, Dublin, 7
}

THIS is the case report of a patient who presented with spontaneous hypoglycaemia and was found to have multiple myeloma. A recent review of multiple myeloma (Healy, 1968), makes no mention of spontaneous hypoglycaemia among the metabolic disturbances that can occur in patients with this condition.

\section{Case report}

M.H., a 61-year-old spinster who was certified blind, because of congenital chorioiditis and was a known hypertensive for some time, but had received no treatment was admitted to the Mater Misericordiae Hospital on 24 December, 1967. She became confused with left-sided weakness following her midday meal, on that day. Her family doctor who examined her at the time found her blood pressure

\footnotetext{
*Present position and address: Lecturer in Medicine, University College, and St Vincent's, Dublin, 4.
}

was $250 / 120 \mathrm{mmHg}$ and that she had a left hemiparesis with an extensor planter response.

On admission about $4 \mathrm{hr}$ later, she was lucid and able to carry on an intelligent conversation, but was confused as to events from mid-day onwards. Vital signs on admission of this obese patient were temperature $98^{\circ} \mathrm{F}$, pulse 80 and repiration $20 / \mathrm{min}$. Her blood pressure was $240 / 150 \mathrm{mmHg}$. She had cardiomegaly but no evidence of congestive cardiac failure. Also she had a left strabismus and bilateral extensive chorioidal and retinal atrophy. Her neurological system was intact with bilateral flexor plantar responses.

The impression was that she had a transient cerebrovascular episode related to her hypertension and she was treated initially with reserpine intramuscularly and later methyldopa and debrisoquine $(1.5 \mathrm{~g}$ and $20 \mathrm{mg}$ daily). Her blood pressure responded well to this therapy. 